\title{
Correction to: Bone health care in women with breast cancer
}

\section{Stavroula A. Paschou ${ }^{1} \cdot$ Areti Augoulea $^{1} \cdot$ Irene Lambrinoudaki $^{1}$}

Published online: 22 January 2020

(C) Hellenic Endocrine Society 2020

\section{Correction to: Hormones}

https://doi.org/10.1007/s42000-019-00164-y

The original version of this article, published on 18 December 2019, contained a mistake. An author's name was misspelled.

In the original version the name Stavroula A. Paschou was written with a Greek alpha.

The correct author name reads as follows:

Stavroula A. Paschou

The original article has been corrected.

The online version of the original article can be found at https://doi.org/ 10.1007/s42000-019-00164-y

Irene Lambrinoudaki

ilambrinoudaki@med.uoa.gr

1 Second Department of Obstetrics and Gynaecology, Aretaieio Hospital, Medical School, National and Kapodistrian University of Athens, Athens, Greece 